Methods Purified $C$ difficile toxin A was labelled with Alexa Fluor 488 (toxin $A^{488}$ ) and its biological activity and specificity of fluorescence were confirmed using Vero cells and anti-toxin A antibody, respectively. Peripheral blood mononuclear cells (PBMNCs) were obtained from 20 patients [13 female, 7 male; median age 67 yrs (range 32-96yrs)] with $C$ difficile infection, within 10 days of diarrhoeal onset. For flow cytometry, PBMNCs were incubated on ice in the dark for $1 \mathrm{~h}$ in the absence or presence of toxin $\mathrm{A}^{488}$. After washing, cells were labelled with anti-CD19-ECD (B cell marker) and anti-IgD-PE (to identify antigen-activated IgD-negativecells). PBMNCs were also polyclonally stimulated in vitro for 6 days to induce differentiation of memory B cells to antibody secreting cells (ASCs). Enzyme-linked immunospot (ELISPOT) assays were used to quantify toxin A-specific IgG ASCs and expressed as percentage of total IgG ASCs. Toxin A-specific IgG antibody levels in sera were studied by ELISA. Data are expressed as median (range).

Results Compared to control buffer, a significantly greater proportion of events (flow cytometry) were seen in the CD19-positive, IgD-negative gate in PBMNCs exposed to toxin $A^{488}[0.09 \%(0 \%-$ $0.54 \%)$ vs $0.92 \%(0.09 \%-1.78 \%) ; p<0.001]$. In four patients studied at the same time as flow cytometry, toxin A-specific ASCs were detected by ELISPOT assays $(0.04 \%-2 \%)$. In studies over $4(1-10)$ months after infection, toxin A-specific ASCs were observed [0.33 $(0.07-2.12) \%$. Serum anti-toxin A antibodies were detectable in eight patients at the time of clinical disease and in four patients, the antibody levels increased over the following $6(2-10)$ months.

Conclusion (1) A small population of toxin A-specific, antigen-activated $B$ cells can be detected in the circulation soon after $C$ difficile infection. (2) In addition to circulating antibody, toxin A-specific memory B cells can be detected over many months after $C$ difficile infection. (3) Future studies can investigate the relationship between the development of $B$ cell responses to $C$ difficile toxins and the nature of clinical disease.

Competing interests None declared.

\section{PWE-109 SHOULD CT COLONOSCOPY REPLACE FLEXIBLE SIGMOIDOSCOPY EXAMINATION AS FIRST LINE INVESTIGATION IN PATIENTS WITH SUSPECTED COLORECTAL CANCER?}

doi:10.1136/gutjnl-2012-302514d.109

${ }^{1} \mathrm{U} D$ Simoniuk, ${ }^{2} \mathrm{~A}$ Milewska, ${ }^{1} \mathrm{C}$ Kirre. ${ }^{1}$ Department of Gastroenterology, Southport Hospital, Southport, UK; ${ }^{2}$ Medical University of Bilaystok, Bialystok, Poland

Introduction It is recommended that all patients undergoing barium enema have a flexible sigmoidoscopy to exclude disease in rectum and sigmoid colon. With the introduction of CT colonoscopy (CT colon) is sigmoidoscopy still required for the investigation of patients with suspected colorectal cancer (CRC). ${ }^{1}$ We reviewed CT colon and flexible sigmoidoscopy results in our patients who underwent both procedures with a view to answering this question. Methods The findings of CT colon in 520 consecutive patients were reviewed by a GI radiologist blinded to the findings at flexible sidmoidoscopy. The mean age of the population was 65 years old. Patients with inadequate bowel preparation for flexible sigmoidoscopy, colonoscopy, polypectomy, abnormal MRI or CT colon as first line investigation and more than 6 months period between CT colon and flexible sigmoidoscopy were excluded. Statistical analysis was performed with $\chi^{2}$ and Fisher test.

Results 332 patients were excluded for the reasons stated above. 188 patients were analysed. In $88 \%$ of these there was concordance between the results of CT colon and flexible sigmoidoscopy. Statistically significant $(p<0.001)$ association was shown between detecting cancer by flexible sigmoidoscopy vs CT colon. The sensitivity and specificity of flexible sigmoidoscopy was $74 \%$ and $99 \%$ respectively [ppv-0.93, npv-0.94]. Flexible sigmoidoscopy did not identify six cancers which were revealed on CT colon. CT colon did not detect two cancers due to collapse colon and further investigation was recommended. There was statistical significant $(p<0.05)$ association between bowel symptoms such as PR bleeding and iron deficiency anaemia and the diagnosis of bowel cancer in patients undergoing flexible sigmoidoscopy.

Conclusion CT colon should replace flexible sigmoidoscopy especially in elderly patients as first line investigation. Flexible sigmoidoscopy still has a role in those patients who found on CT colon to have a collapse left colon.

Competing interests None declared.

\section{REFERENCE}

1. NICE clinical guideline. "The Diagnosis and Management of Colorectal Cancer". Issued: November 2011.

\section{PWE-110 WHAT IS THE SIGNIFICANCE OF "DIMINUTIVE" COLONIC POLYPS IN PATIENTS SCREENED FOR COLON CANCER AFTER A POSITIVE FAECAL HUMAN HAEMOGLOBIN TEST (FHH)?}

doi:10.1136/gutjnl-2012-302514d.110

V Mahesh, ${ }^{*}$ A Lim, K Pietris, J Argyrides. Department of Gastroenterology, Royal Adelaide Hospital, Adelaide, Australia

Introduction By definition "diminutive" colonic polyps are $\leq 5 \mathrm{~mm}$ in size. Improvements in optical imaging modalities and image resolution coupled with increased operator awareness have resulted in a higher detection rates at colonoscopy. Although such polyps are low risk for malignancy, studies suggest that high grade dysplasia may occur up to a rate of $4 \%$ in these polyps. There is an ongoing debate regarding the natural history and prognostic relevance of these polyps including suggestions for non-resection/to resect and discard. Aim To analyse the significance of "Diminutive" colonic polyps in patients screened for colon cancer after a positive Faecal Human Haemoglobin test.

Methods Patients referred to a single tertiary institution in South Australia for screening colonoscopy after detection of a positive FHH. All patients referred between 2007 and 2010 were included in this retrospective study. The patients were referred either through the National Bowel Cancer Screening Program (NBCSP) or through GP initiated screening. Polyps were measured after the tissue was placed in formalin (histological size). For each colonoscopy the predetermined aim was to resect all polyps and assess histologically. Results A total of 384 patients had colonoscopy. NBCSP referred $(\mathrm{n}=173,45 \%)$ and GP initiated FOBT ( $\mathrm{n}=211,55 \%) .228 \mathrm{M}: 156 \mathrm{~F}$ with mean age of $61.8 \pm 0.6 \mathrm{yrs}, 305$ polyps in total. Majority of the polyps $(57 \%, \mathrm{n}=173)$ were $\leq 5 \mathrm{~mm}$ compared with $6-9 \mathrm{~mm}(23 \%$,

Abstract PWE-110 Table 1 Histology of polyps $\leq 5 \mathrm{~mm}$ in size

\begin{tabular}{lrl}
\hline Tubular adenoma- low grade dysplasia & 86 & $48 \%$ \\
Tubular adenoma-high grade dysplasia & 1 & $0.6 \%$ \\
Serrated adenoma & 8 & $4.6 \%$ \\
Tubulovillous adenoma-low grade dysplasia & 10 & $5.8 \%$ \\
Tubulovillous adenoma-high grade dysplasia & 0 & $0 \%$ \\
Villous adenoma-low grade dysplasia & 1 & $0.6 \%$ \\
Villous adenoma-high grade dysplasia & 0 & $0 \%$ \\
Adenocarcinoma & 0 & $0 \%$ \\
Hyperplastic polyps & 66 & $38.1 \%$ \\
Total & 173 & \\
\hline
\end{tabular}

